\title{
ADDITIONAL EVIDENCE FOR THE LOCATION OF THE $\alpha$-NEURAMINIDASE GENE ON CHROMOSOME 6
}

\author{
Takahiko OoHIRA, ${ }^{1}$ Michihiro C. Yoshida, ${ }^{2}$ and Ichiro MATsuda ${ }^{1}$ \\ ${ }^{3}$ Department of Pediatrics, Kumamoto University Medical School, Kumamoto, \\ Japan, and ${ }^{2}$ Chromosome Research Unit, Faculty of Science, \\ Hokkaido University, Sappro, Japan
}

Observations in a patient with combined deficiency of $\alpha$-neuraminidase and 21-hydroxylase whose parents are consanguineous indicate that $\alpha$-neuraminidase deficiency, similar to 21-hydroxylase deficiency, segregate with HLA-complex (Oohira et al., 1985), and that the $\alpha$-neuraminidase locus is on chromosome 6, probably close to the 21-hydroxylase locus and the HLA complex loci. In order to obtain additional evidence for the location of the $\alpha$-neuraminidase gene on chromosome 6 , we measured $\alpha$-neuraminidase activity in somatic cell hybrid clones established from fusion between human HE2144 diploid fibroblasts and mouse B82 cells originated from the L strain.

The hybrid clone, laD5, retained human chromosome 6 , while the 1a-13 clone loss chromosome 6. The constitution of the other human chromosomes in these hybrid clones was mostly the same (unpublished data). Since separation of human and mouse $\alpha$-neuraminidase by electrophoresis is difficult because of unstability of the enzyme during the procedure, quantitative analysis of the enzyme was performed. $\alpha$-Neuraminidase activity was measured using 4-methylumbelliferyl- $N$-acetyl neuraminic acid (Nakarai Chemicals Ltd., Kyoto) (Potier et al., 1979).

The results obtained are shown in Table 1. It is demonstrated that a hybrid clone retaining human chromosome 6 had significantly increased neuraminidase activity compared to that of the mouse parental cell line $(\mathrm{p}<0.005)$ and that of hybrid cells missing human chromosome $6(\mathrm{p}<0.05)$, though the activity was found to be considerably lower than that in original human fibroblasts. This observation may indicate that there is species specific control mechanism for full expression of human $\alpha$-neuraminidase gene in human cells which is impared in hybrids containing mouse genome. Although the mechanism of the reduced expression of the human $\alpha$-neuraminidase gene in mouse cell is obscure, the possibility that the $\alpha$-neuraminidase locus is on chromosome 6 is supported by this observation.

Received June 14, 1986; Accepted June 28, 1986 
Table 1. $\alpha$-Neuraminidase activity of mouse and human skin fibroblasts and hybrid clones.

\begin{tabular}{lcc}
\hline Cells & $\begin{array}{c}a \text {-Neuraminidase activity } \\
\text { (nmol/mg protein/hr) }\end{array}$ \\
\hline Mouse parental fibroblast $\quad(\mathrm{n}=13)$ & $1.08 \pm 0.38^{\mathrm{a}}$ \\
Hybrid (laD5) retaining human chromosome $6 \quad(\mathrm{n}=7)$ & $3.20 \pm 1.96$ \\
Hybrid (la-13) missing human chromosome $6 \quad(\mathrm{n}=9)$ & $1.37 \pm 0.83^{\mathrm{b}}$ \\
Human skin (HE2144) fibroblast $\quad(\mathrm{n}=13)$ & $37.1 \pm 5.6^{\mathrm{c}}$ \\
\hline
\end{tabular}

Compared with hybrids retaining human chromosome 6: ${ }^{\mathrm{a}} \mathrm{p}<0.005,{ }^{\mathrm{b}} \mathrm{p}<0.05,{ }^{\mathrm{c}} \mathrm{p}<0.001$.

\section{REFERENCES}

Oohira, T., Nagata, N., Akaboshi, I., Matsuda, I., and Naito, S. 1985. The infantile form of sialidosis type II associated with congenital adrenal hyperplasis: Possible linkage between HLA and the neuraminidase deficiency gene. Hum. Genet. 70: 341-343.

Potier, M., Mameli, L., Bélisle, M., Dallarie, L., and Melançon, S.B. 1979. Fluorometric assay of neuraminidase with a sodium (4-methylumbelliferyl- $\alpha-\mathrm{D}-N$-acetylneuraminate) substrate. Anal. Biochem. 94: 287-296 